\title{
ON SOME NUMERICAL TECHNIQUES FOR STRESS-CONSTRAINED CONTINUA
}

\author{
MASSIMILIANO LUCCHESI, BARBARA PINTUCCHI \& NICOLA ZANI \\ Department of Civil and Environmental Engineering (DICeA), University of Florence, Italy
}

\begin{abstract}
A numerical method is described for the semi-explicit solution of the constitutive equation of an orthotropic non-linear elastic material with various constraints on the stress. Each constraint forces the stress to belong to a closed and convex cone in the space of second order symmetric tensors, and via the choice of appropriate values cones vertex is possible to assign different strength characteristic in different directions. The constitutive equation thus formulated allows to model masonry with very general textures. However application to problems of equilibrium and evolution requires some numerical expedients which are described in detail in this paper. The equation, implemented in the MADY finite element code, has been used to examine how the masonry panel strength changes as a function of traction directions. The results have been compared with the analougous obtained modelling the masonry at the micro scale.
\end{abstract}

Keywords: orthotropic materials, masonry panels.

\section{INTRODUCTION}

A constitutive model for masonry materials requires accounting for its poor tensile strength. This need led to the study of the masonry-like material [1], which in its original formulation imposes the constraint on the stress $\boldsymbol{T}$ of being semi-defined negative. The equation was then generalized by also imposing a limit on compressive and shear strength [2], [3]. Each of these constraints requires $\boldsymbol{T}$ to belong to a specific closed and convex cone, whose intersection is the stress range $\mathcal{K}$. Having assigned a strain tensor $E$ and the symmetric and positivedefinite tensor $\mathbb{C}$ of the elastic moduli, the stress $\boldsymbol{T}$ is obtained by projecting $\mathbb{C} E$ onto $\mathcal{K}$, with respect to a suitable scalar product. In other words, it is required that the inelastic strain $E^{a}=E-\mathbb{C}^{-1} T$ belongs to the normal cone of $\mathcal{K}$ in $\boldsymbol{T}$. The existence and uniqueness of the projection follows by the minimum norm theorem and the result is a hyperelastic material which has been defined normal-elastic material.

In order to apply this constitutive equation to engineering problems, it is necessary to resort to numerical analysis. This in turn required that the constitutive equation be explicitly solved and the derivative of the stress with respect to the strain explicitly calculated [4]. With the constraints on the stress that were taken into account, $\mathcal{K}$ resulted to be a spherical set, i.e. such that $\mathcal{K}=Q \mathcal{K} Q^{T}$, for each rotation $Q$, and moreover $\mathbb{C}$ was hypothesized to be an isotropic tensor. The first of these properties implies the coaxiality between the stress and the inelastic strain, and the second (if the first is guaranteed) implies the coaxiality between the stress and the strain. The fact that $T, E$ and $E^{a}$ are all coaxial tensors allowed to solve the constitutive equation in their common characteristic space, simplifying the problem. This constitutive equation has been applied to the study of numerous monuments and may be effective for certain buildings where the texture and the properties of the masonry cannot be easily evaluable. On the other hand, for different applications a model that takes into account the different properties of the material in different directions is certainly more realistic [5], [6]. For this reason in [7] the equation has been generalized allowing the tensor 
of the elastic moduli to be orthotropic. Consequently, the coaxiality between $\boldsymbol{T}$ and $\boldsymbol{E}$ has been lost, but that between $\boldsymbol{T}$ and $\boldsymbol{E}^{a}$ is maintained.

In [8], the material has been supposed to have different strength characteristics in different directions, a circumstance that can often occur due to the constructive techniques and to material's damage process. In such a case, the stress range $\mathcal{K}$ is made by the intersection of several closed and convex cones each of which has as its vertex a tensor that is not necessarily spherical. Thus, the coaxiality between $\boldsymbol{T}$ and $\boldsymbol{E}^{a}$ is lost. Despite the greater complexity that must be tackled, the strain space is always naturally divided into a finite number of regions and $\boldsymbol{E}$ belong to one of these determines the solution of the constitutive equation. Thanks to this property, albeit with some expedient, it is possible to obtain an "almost explicit" solution of the constitutive equation. However, this generalization requires new numerical procedures, mainly because it is not possible to determine a priori the region to which the deformation belongs, and therefore it is necessary to proceed by trials and errors.

In this paper, the numerical techniques that allow to apply this constitutive equation to equilibrium and evolution problems is described in detail. Then the equation, implemented in the finite element code MADY, is used to solve same test case. In particular, a panel with a typical texture is considered and analyzed at the microscale by subjecting it to a tensile force that is applied in five different directions and increased until the collapse. Subsequently, a "homogeneous" panel with a tensile strength deduced from the previous test is subjected to the same loads. It is evidenced how the texture can be well modelled by a suitable choice of the vertices of $\mathcal{K}$.

\section{NOTATIONS AND BACKGROUND}

Let Sym be the space of the symmetric second order tensor with the inner product $\boldsymbol{A} \cdot \boldsymbol{B}=\operatorname{tr}(\boldsymbol{A} \boldsymbol{B})$, and the corresponding Euclidean norm \|\| . The energetic inner product $(\boldsymbol{A}, \boldsymbol{B})_{\boldsymbol{E}}=\boldsymbol{A} \cdot \mathbb{C}^{-1} \boldsymbol{B}$ and the corresponding norm denoted by \|\|$\|_{\boldsymbol{E}}$ will also be considered. Moreover, if $\mathcal{K} \subset$ Sym is the non empty, closed and convex set made by all the admissible stresses, for each $T \in \partial \mathcal{K}$ (the boundary of $\mathcal{K}$ ), the normal cone of $\mathcal{K}$ at $\boldsymbol{E}$ is denoted by $\mathcal{N}(\mathcal{K}, T)$.

Assigned a strain tensor $\boldsymbol{E}$, there exists only one element $\boldsymbol{T} \in \mathcal{K}$ having the minimum energy distance from $\mathbb{C} \boldsymbol{E}$, i.e. such that

$$
\|\mathbb{C} \boldsymbol{E}-\boldsymbol{T}\|_{\boldsymbol{E}}=\min _{\boldsymbol{S} \in \mathcal{K}}\|\mathbb{C} \boldsymbol{E}-\boldsymbol{S}\|_{\boldsymbol{E}} .
$$

This is equivalent to saying that $\boldsymbol{T}$ is the projection of $\mathbb{C E}$ onto $\mathcal{K}$, with respect to the energetic norm, i.e. $\boldsymbol{E}-\mathbb{C}^{-1} \boldsymbol{T}$ belongs to $\mathcal{N}(\mathcal{K}, \boldsymbol{T})$.

Relation $\boldsymbol{T}=\hat{\boldsymbol{T}}(\boldsymbol{E})$ defines the constitutive equation of a non linear hyperelastic material that is termed normal elastic material [1]. Once set

$$
\boldsymbol{E}^{e}=\mathbb{C}^{-1} \boldsymbol{T}, \quad \boldsymbol{E}^{a}=\boldsymbol{E}-\mathbb{C}^{-1} \boldsymbol{T},
$$

it turns out $\boldsymbol{E}=\boldsymbol{E}^{e}+\boldsymbol{E}^{a} ; \boldsymbol{E}^{e}$ and $\boldsymbol{E}^{a}$ are said, elastic and inelastic part of the deformation, respectively In particular, if $\boldsymbol{T}$ is a regular point of $\partial \mathcal{K}$ and $\boldsymbol{N}(\boldsymbol{T})$ is the corresponding unit outward normal, then

$$
E^{a}=\alpha \boldsymbol{N}(\boldsymbol{T}), \quad \alpha \geq 0,
$$

holds. Note that if $\mathcal{K}$ is a spherical tensor then $\boldsymbol{N}(\boldsymbol{T})$ is an isotropic function of $\boldsymbol{T}$ and then $\boldsymbol{T}$ and $\boldsymbol{E}^{a}$ have the same characteristic space so that $\boldsymbol{T} \boldsymbol{E}^{a}=\boldsymbol{E}^{a} \boldsymbol{T}$.

From eqn (2)

$$
\mathbb{C} \boldsymbol{E}-\boldsymbol{T}=\alpha \mathbb{C} \boldsymbol{N}(\boldsymbol{T})
$$


follows and then, with a few substitutions,

$$
\boldsymbol{T}=\mathbb{C} \boldsymbol{E}-\frac{(\mathbb{C} E-T) \cdot \boldsymbol{N}}{\boldsymbol{N} \cdot \mathbb{C} \boldsymbol{N}} \mathbb{C}=\mathbb{C} \boldsymbol{E}-\frac{(\mathbb{C} \boldsymbol{N} \otimes \boldsymbol{N})}{\boldsymbol{N} \cdot \mathbb{C N}}(\mathbb{C} \boldsymbol{E}-\boldsymbol{T})
$$

is obtained.

Let be $\mathcal{E}=\mathbb{C}^{-1}(\mathcal{K})$; if $\boldsymbol{T}=\hat{\boldsymbol{T}}(\boldsymbol{E}) \in \partial \mathcal{K}$ then $\boldsymbol{E} \in \partial \mathcal{E}$ and, if $\boldsymbol{T}$ is a regular point of $\partial \mathcal{K}$ also $\boldsymbol{E}$ is a regular point of $\partial \mathcal{E}$, with outward unit normal $\boldsymbol{M}(\boldsymbol{E})=$ $\mathbb{C} \boldsymbol{N}(\hat{\boldsymbol{T}}(\boldsymbol{E})) /\|\mathbb{C} \boldsymbol{N}(\hat{\boldsymbol{T}}(\boldsymbol{E}))\|$.

A convex cone with vertex $\boldsymbol{V}$ is a non-empty, closed set $\mathcal{C}$ containing the origin $\mathbf{0}$ of Sym, such that

$$
\boldsymbol{V}+a(\boldsymbol{S}-\boldsymbol{V})+b(\boldsymbol{T}-\boldsymbol{V}) \in \mathcal{C} \text {, for each } \boldsymbol{S}, \boldsymbol{T} \in \mathcal{C} \text { and for each } a \geq 0 \text { and } b \geq 0 .
$$

Proceeding in a similar way to what done in [9], it can be verified that for this set the normal cone $\mathcal{N}(\mathcal{C}, T)$ is made up of all the elements $\boldsymbol{A} \in$ Sym such that

$$
\text { (i) }(\boldsymbol{T}-\boldsymbol{V}) \cdot \boldsymbol{A}=0, \quad \text { (ii) }(\boldsymbol{S}-\boldsymbol{V}) \cdot \boldsymbol{A} \leq 0 \text {, for each } \boldsymbol{S} \in \mathcal{C} .
$$

Let $\boldsymbol{T} \in \partial \mathcal{C}$ be a regular point and $\boldsymbol{N}(\boldsymbol{T})$ the corresponding outward unit normal. Then, in view of eqn $(6)_{1}$ it holds

$$
(\boldsymbol{T}-\boldsymbol{V}) \cdot \boldsymbol{N}(\boldsymbol{T})=0
$$

This implies

$$
(\mathbb{C} \boldsymbol{E}-\boldsymbol{T}) \cdot \boldsymbol{N}(\boldsymbol{T})=(\mathbb{C} \boldsymbol{E}-\boldsymbol{T}+\boldsymbol{T}-\boldsymbol{V}) \cdot \boldsymbol{N}(\boldsymbol{T})=(\mathbb{C} \boldsymbol{E}-\boldsymbol{V}) \cdot \boldsymbol{N}(\boldsymbol{T}),
$$

and then from eqn (4) it follows

$$
\boldsymbol{T}=\mathbb{C} \boldsymbol{E}-\frac{(\mathbb{C} \boldsymbol{N} \otimes \boldsymbol{N})}{\boldsymbol{N} \cdot \mathbb{C} N}(\mathbb{C} \boldsymbol{E}-\boldsymbol{V})
$$

which is more convenient relation than eqn (4) because in applications $\boldsymbol{V}$ is known.

\section{MATERIALS WITH LIMITED TENSILE, COMPRESSIVE AND SHEAR STRENGTH}

The Let $\mathrm{Sym}^{-}$and $\mathrm{Sym}^{+}$the subsets of Sym consisting of the negative and positive semi definite tensors, respectively, and let $\boldsymbol{T}_{t}, \boldsymbol{T}_{c}$ and $\boldsymbol{T}_{s}$ be positive semi definite tensors. A material is said to have limited tensile, compressive and shear strength if the stress is constrained to belonging to the closed and convex set $\mathcal{K}$ which is made by the intersection of the three cones

$$
\begin{aligned}
& \mathcal{T}=\left\{\boldsymbol{T} \in \text { Sym }: \operatorname{tr}\left(\boldsymbol{T}-\boldsymbol{T}_{t}\right) \leq 0, \quad \operatorname{det}\left(\boldsymbol{T}-\boldsymbol{T}_{t}\right) \geq 0\right\}, \\
& \mathcal{C}=\left\{\boldsymbol{T} \in \text { Sym }: \operatorname{tr}\left(\boldsymbol{T}+\boldsymbol{T}_{c}\right) \geq 0, \quad \operatorname{det}\left(\boldsymbol{T}+\boldsymbol{T}_{c}\right) \geq 0\right\}
\end{aligned}
$$

and

$$
\mathcal{S}=\left\{\boldsymbol{T} \in \operatorname{Sym}:\left\|\boldsymbol{T}-\boldsymbol{T}_{s}\right\|^{2}-\frac{1+\sin ^{2} \phi}{2}\left(\operatorname{tr}\left(\boldsymbol{T}-\boldsymbol{T}_{s}\right)\right)^{2} \leq 0, \operatorname{tr}\left(\boldsymbol{T}-\boldsymbol{T}_{s}\right) \leq 0\right\}
$$

with vertex $\boldsymbol{T}_{t}, \boldsymbol{T}_{c}$ and $\boldsymbol{T}_{s}$, respectively, and whose boundary is made up, in addition to $\left\{\boldsymbol{T}_{t}\right\},\left\{\boldsymbol{T}_{c}\right\}$ and $\boldsymbol{T}_{s}$, of the sets

$$
\left\{\boldsymbol{T} \in \operatorname{Sym}: \operatorname{tr}\left(\boldsymbol{T}-\boldsymbol{T}_{t}\right)<0, \quad \operatorname{det}\left(\boldsymbol{T}-\boldsymbol{T}_{t}\right)=0\right\},
$$



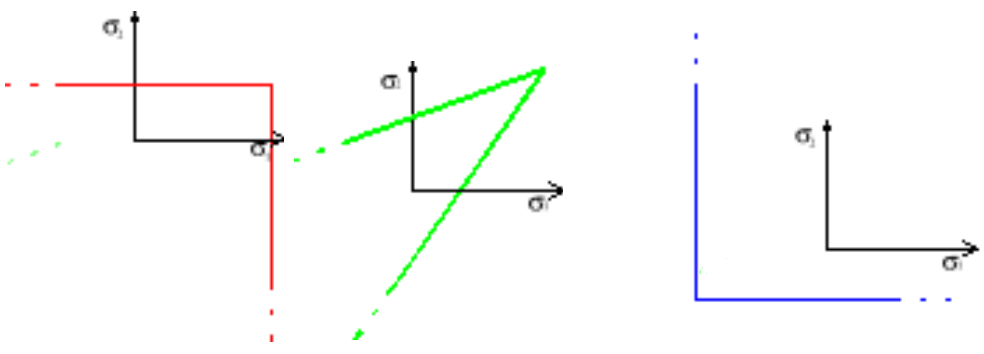

Figure 1: The stress constraints.

$$
\left\{\boldsymbol{T} \in \operatorname{Sym}: \operatorname{tr}\left(\boldsymbol{T}+\boldsymbol{T}_{c}\right)>0, \quad \operatorname{det}\left(\boldsymbol{T}+\boldsymbol{T}_{c}\right)=0\right\}
$$

and

$$
\left\{\boldsymbol{T} \in \operatorname{Sym}: 2\left\|\boldsymbol{T}-\boldsymbol{T}_{s}\right\|^{2}-\left(1+\sin ^{2} \phi\right) \operatorname{tr}\left(\boldsymbol{T}-\boldsymbol{T}_{s}\right)^{2}=0, \quad \operatorname{tr}\left(\boldsymbol{T}-\boldsymbol{T}_{s}\right)<0\right\} .
$$

The three cones are shown in Fig. 1, with different colors because they are represented in the characteristic spaces of $\boldsymbol{T}-\boldsymbol{T}_{t}, \boldsymbol{T}+\boldsymbol{T}_{c}$ and $\boldsymbol{T}-\boldsymbol{T}_{s}$, which in general are different from each other. It comes down to the usual case when $\boldsymbol{T}_{t}, \boldsymbol{T}_{c}$ and $\boldsymbol{T}_{s}$ are spherical tensors, and the coaxiality of $\boldsymbol{T}, \boldsymbol{T}_{t}, \boldsymbol{T}_{c}$ and $\boldsymbol{T}_{s}$ allows to represent $\mathcal{K}$ in the characteristic space of $\boldsymbol{T}$, as shown in Fig. 2(a).

\section{DETERMINATION OF THE STRESS}

Let the orthonormal vectors $\boldsymbol{e}_{1}$ and $\boldsymbol{e}_{2}$ define the symmetry directions of a plane orthotropic body [10] which are assumed as refence system. The elasticity tensor is

$$
\mathbb{C}=\left(\begin{array}{ccc}
C_{1111} & C_{1122} & 0 \\
C_{1112} & C_{2222} & 0 \\
0 & 0 & C_{2323}
\end{array}\right)
$$

where

$$
\begin{gathered}
C_{1111}=\frac{E_{11}}{1-\nu_{12} \nu_{21}}, \quad C_{1122}=\frac{\nu_{12} E_{22}}{1-\nu_{12} \nu_{21}}=\frac{\nu_{21} E_{11}}{1-\nu_{12} \nu_{21}}, \\
C_{2222}=\frac{E_{22}}{1-\nu_{12} \nu_{21}}, \quad C_{2323}=2 G,
\end{gathered}
$$

with $E_{11}, E_{22}$ and $\nu_{12}, \nu_{21}$ the corresponding Young and Poisson moduli, respectively.

By denoting

$$
E_{22}=\beta E_{11}, \quad \nu_{21}=\beta \nu_{12}, \quad 2 G=\frac{\phi E_{11}}{1-\beta \nu_{12}^{2}}
$$

and writing $E$ for $E_{11}$ and $\nu$ for $\nu_{12}$, it is obtained

$$
\mathbb{C}=\frac{E}{1-\beta \nu^{2}}\left(\begin{array}{ccc}
1 & \beta \nu & 0 \\
\beta \nu & \beta & 0 \\
0 & 0 & \phi
\end{array}\right)
$$

and

$$
\mathbb{C}^{-1}=\frac{1}{E}\left(\begin{array}{ccc}
1 & -\nu & 0 \\
-\nu & 1 / \beta & 0 \\
0 & 0 & \left(1-\beta \nu^{2}\right) / \phi
\end{array}\right)
$$


In the particular case of $\beta=1$ and $\phi=1-\nu$, the material is isotropic. Let

$$
\boldsymbol{E}=\epsilon_{11} \boldsymbol{e}_{1} \otimes \boldsymbol{e}_{1}+\epsilon_{22} \boldsymbol{e}_{2} \otimes \boldsymbol{e}_{2}+\epsilon_{12}\left(\boldsymbol{e}_{1} \otimes \boldsymbol{e}_{2}+\boldsymbol{e}_{2} \otimes \boldsymbol{e}_{1}\right)
$$

be the assigned strain tensor so that

$$
\begin{gathered}
\mathbb{C} \boldsymbol{E}=\frac{E}{1-\beta \nu^{2}}\left(\left(\epsilon_{11}+\beta \nu \epsilon_{22}\right) \boldsymbol{e}_{1} \otimes \boldsymbol{e}_{1}+\beta\left(\epsilon_{22}+\nu \epsilon_{11}\right) \boldsymbol{e}_{2} \otimes \boldsymbol{e}_{2}\right. \\
\left.+\phi \epsilon_{12}\left(\boldsymbol{e}_{1} \otimes \boldsymbol{e}_{2}+\boldsymbol{e}_{2} \otimes \boldsymbol{e}_{1}\right)\right) .
\end{gathered}
$$

In order to solve the constitutive equation a semi explicit procedure has been developed. All constraints are expressed in the reference system.

Let $\mathcal{T}$ be the traction cone with vertex

$$
\boldsymbol{T}_{t}=\sigma_{t x} \mathbf{e}_{1} \otimes \mathbf{e}_{1}+\sigma_{t y} \mathbf{e}_{2} \otimes \mathbf{e}_{2}+\tau_{t}\left(\mathbf{e}_{1} \otimes \mathbf{e}_{2}+\mathbf{e}_{2} \otimes \mathbf{e}_{1}\right) .
$$

The eigenvalues $\left(\sigma_{t_{1}}, \sigma_{t_{2}}\right)$ are the values of the tensile strength referred to the corresponding principal directions $\left(\boldsymbol{e}_{t_{1}}, \boldsymbol{e}_{t_{2}}\right)$. In the characteristic space, the cone can be represented as a plane region as shown in Fig. 1(a).

Analougously, the shear cone $\mathcal{S}$ has vertex

$$
\boldsymbol{T}_{s}=\frac{\tau_{0 x}}{m} \boldsymbol{e}_{1} \otimes \boldsymbol{e}_{1}+\frac{\tau_{0 y}}{m} \boldsymbol{e}_{2} \otimes \boldsymbol{e}_{2}+\frac{\tau_{s}}{m}\left(\mathbf{e}_{1} \otimes \mathbf{e}_{2}+\mathbf{e}_{2} \otimes \mathbf{e}_{1}\right) .
$$

Its eigenvalues $\left(\frac{\tau_{0_{1}}}{m}, \frac{\tau_{0_{2}}}{m}\right)$ are the ratio between coesion and $m=\tan \phi$, referred to the principal directions $\left(\boldsymbol{e}_{s_{1}}, \boldsymbol{e}_{s_{2}}\right)$. In this characteristic space, such constraint can be represented as a plane region, as shown in Fig. 1(b).

Lastly, the compression cone $\mathcal{C}$ has vertex

$$
\boldsymbol{T}_{c}=\sigma_{c x} \mathbf{e}_{1} \otimes \mathbf{e}_{1}+\sigma_{c y} \mathbf{e}_{2} \otimes \mathbf{e}_{2}+\tau_{c}\left(\mathbf{e}_{1} \otimes \mathbf{e}_{2}+\mathbf{e}_{2} \otimes \mathbf{e}_{1}\right),
$$

whose eigenvalues are the value of compressive strength $\left(\sigma_{c_{1}}, \sigma_{c_{2}}\right)$ referred to its principal directions $\left(\boldsymbol{e}_{c_{1}}, \boldsymbol{e}_{c_{2}}\right)$. In its characteristic space, $\mathcal{C}$ can be represented as a plane region as given by Fig. 1(c).

If the vertices of the cones are spherical tensors and $\mathbb{C}$ is isotropic, $\mathcal{K}$ is a spherical set (i.e. $\boldsymbol{T} \in \mathcal{K} \Leftrightarrow \boldsymbol{Q} \boldsymbol{T} \boldsymbol{Q}^{T} \in \mathcal{K}$ for each rotation $\boldsymbol{Q}$ ). Then, the characteristic spaces of $\boldsymbol{T}-\boldsymbol{T}_{t}$, $\boldsymbol{T}-\boldsymbol{T}_{s}$ and $\boldsymbol{T}+\boldsymbol{T}_{c}$ coincide so that $\mathcal{K}$ (the intersection among the three cones) and $\boldsymbol{E}^{a}$ can be represented, in the common characteristic space as a plane domain (Fig. 2(a)).

If the vertices of the cones are not spherical tensors, a plane representation of $\mathcal{K}$ does not exist; however, the 2D pseudo-representation depicted in Fig. 2(b) is useful, as it allows an easier determination of the normals to the edges of $\partial \mathcal{K}$. The use of different colours underlines that such representation refers simultaneously to different reference systems.

When the constraints act simultaneously, $\mathcal{K}$ depends on $\boldsymbol{T}_{t}, \boldsymbol{T}_{s}$ and $\boldsymbol{T}_{c}$, and three different situations can occur.

Case 1: The traction constraint is ineffective (Fig. 3), as $\boldsymbol{T}_{t}$ is outside the shear cone i.e.

$$
2\left\|\boldsymbol{T}_{t}-\boldsymbol{T}_{s}\right\|^{2}-\left(1+\sin ^{2} \phi\right) \operatorname{tr}\left(\boldsymbol{T}_{t}-\boldsymbol{T}_{s}\right)^{2}>0, \quad \operatorname{tr}\left(\boldsymbol{T}_{t}-\boldsymbol{T}_{s}\right)>0 .
$$

Case 2: All the constraints are effective (Fig. 4), when $\boldsymbol{T}_{t}$ belong to shear cone

$$
2\left\|\boldsymbol{T}_{t}-\boldsymbol{T}_{s}\right\|^{2}-\left(1+\sin ^{2} \phi\right) \operatorname{tr}\left(\boldsymbol{T}_{t}-\boldsymbol{T}_{s}\right)^{2} \leq 0, \quad \operatorname{tr}\left(\boldsymbol{T}_{t}-\boldsymbol{T}_{s}\right) \leq 0 .
$$




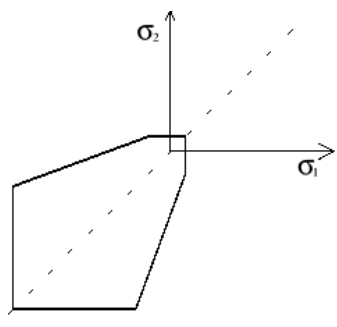

a

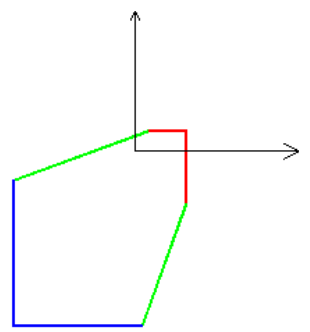

b

Figure 2: The admissible domain.
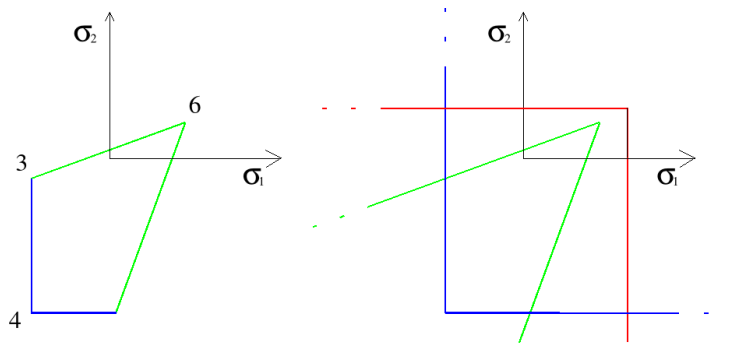

Figure 3: Case 1.
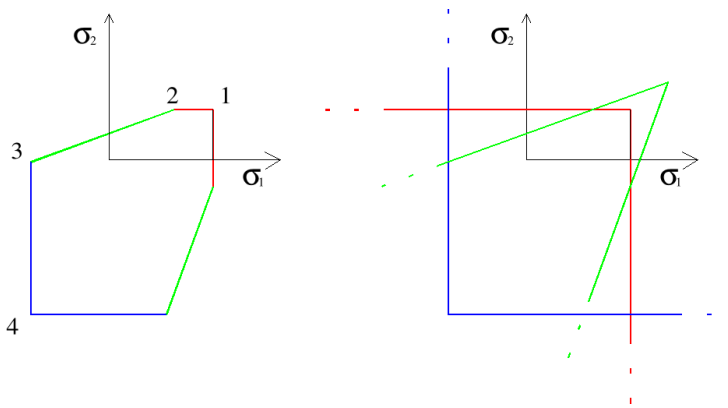

Figure 4: Case 2.

Case 3: the shear constraint is ineffective (Fig. 5), as the intersection between the cones $\mathcal{T}$ and $\mathcal{C}$ is entirely contained in the cone $\mathcal{S}$.

For the sake of example, the semi-explicit procedure to determine the solution of the constitutive equation is given with reference to Case 2.

Let us denote $\mathcal{R}_{0}$ as the interior of $\mathcal{K}$ and $\mathcal{R}_{1}, \mathcal{R}_{12}, \mathcal{R}_{2}, \mathcal{R}_{23}, \mathcal{R}_{3}, \mathcal{R}_{34}$ and $\mathcal{R}_{4}$ the regions that define a partition of $\partial \mathcal{K}$ :

$$
\begin{array}{r}
\mathcal{R}_{0}=\left\{\boldsymbol{T} \in \text { Sym }: \operatorname{det}\left(\boldsymbol{T}-\boldsymbol{T}_{t}\right) \geq 0, \operatorname{tr}\left(\boldsymbol{T}-\boldsymbol{T}_{t}\right) \leq 0,\right. \\
2\left\|\boldsymbol{T}-\boldsymbol{T}_{s}\right\|^{2}-\left(1+\sin ^{2} \phi\right) \operatorname{tr}\left(\boldsymbol{T}-\boldsymbol{T}_{s}\right)^{2} \leq 0, \operatorname{tr}\left(\boldsymbol{T}-\boldsymbol{T}_{s}\right) \leq 0 \\
\left.\operatorname{det}\left(\boldsymbol{T}+\boldsymbol{T}_{c}\right) \geq 0, \operatorname{tr}\left(\boldsymbol{T}+\boldsymbol{T}_{c}\right) \geq 0\right\},
\end{array}
$$



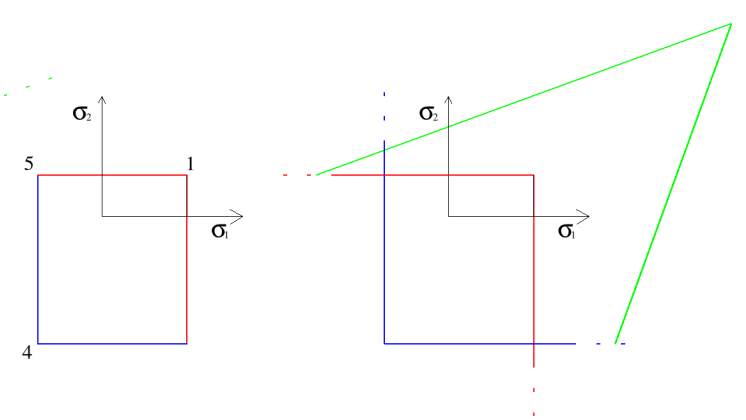

Figure 5: Case 3.

$$
\begin{aligned}
& \mathcal{R}_{1}=\left\{\boldsymbol{T}_{t}\right\} \\
& \mathcal{R}_{12}=\left\{\boldsymbol{T} \in \operatorname{Sym}: \operatorname{det}\left(\boldsymbol{T}-\boldsymbol{T}_{t}\right)=0, \operatorname{tr}\left(\boldsymbol{T}-\boldsymbol{T}_{t}\right) \leq 0,\right. \\
& 2\left\|\boldsymbol{T}-\boldsymbol{T}_{s}\right\|^{2}-\left(1+\sin ^{2} \phi\right) \operatorname{tr}\left(\boldsymbol{T}-\boldsymbol{T}_{s}\right)^{2} \leq 0, \operatorname{tr}\left(\boldsymbol{T}-\boldsymbol{T}_{s}\right) \leq 0 \\
& \left.\operatorname{det}\left(\boldsymbol{T}+\boldsymbol{T}_{c}\right) \geq 0, \operatorname{tr}\left(\boldsymbol{T}+\boldsymbol{T}_{c}\right) \geq 0\right\}, \\
& \mathcal{R}_{2}=\left\{\boldsymbol{T} \in \operatorname{Sym}: \operatorname{det}\left(\boldsymbol{T}-\boldsymbol{T}_{t}\right)=0, \operatorname{tr}\left(\boldsymbol{T}-\boldsymbol{T}_{t}\right) \leq 0,\right. \\
& 2\left\|\boldsymbol{T}-\boldsymbol{T}_{s}\right\|^{2}-\left(1+\sin ^{2} \phi\right) \operatorname{tr}\left(\boldsymbol{T}-\boldsymbol{T}_{s}\right)^{2}=0, \operatorname{tr}\left(\boldsymbol{T}-\boldsymbol{T}_{s}\right) \leq 0 \\
& \left.\operatorname{det}\left(\boldsymbol{T}+\boldsymbol{T}_{c}\right) \geq 0, \operatorname{tr}\left(\boldsymbol{T}+\boldsymbol{T}_{c}\right) \geq 0\right\}, \\
& \mathcal{R}_{23}=\left\{\boldsymbol{T} \in \operatorname{Sym}: \operatorname{det}\left(\boldsymbol{T}-\boldsymbol{T}_{t}\right) \geq 0, \operatorname{tr}\left(\boldsymbol{T}-\boldsymbol{T}_{t}\right) \leq 0,\right. \\
& 2\left\|\boldsymbol{T}-\boldsymbol{T}_{s}\right\|^{2}-\left(1+\sin ^{2} \phi\right) \operatorname{tr}\left(\boldsymbol{T}-\boldsymbol{T}_{s}\right)^{2}=0, \operatorname{tr}\left(\boldsymbol{T}-\boldsymbol{T}_{s}\right) \leq 0 \\
& \left.\operatorname{det}\left(\boldsymbol{T}+\boldsymbol{T}_{c}\right) \geq 0, \operatorname{tr}\left(\boldsymbol{T}+\boldsymbol{T}_{c}\right) \geq 0\right\}, \\
& \mathcal{R}_{3}=\left\{\boldsymbol{T} \in \operatorname{Sym}: \operatorname{det}\left(\boldsymbol{T}-\boldsymbol{T}_{t}\right) \geq 0, \operatorname{tr}\left(\boldsymbol{T}-\boldsymbol{T}_{t}\right) \leq 0,\right. \\
& 2\left\|\boldsymbol{T}-\boldsymbol{T}_{s}\right\|^{2}-\left(1+\sin ^{2} \phi\right) \operatorname{tr}\left(\boldsymbol{T}-\boldsymbol{T}_{s}\right)^{2}=0, \operatorname{tr}\left(\boldsymbol{T}-\boldsymbol{T}_{s}\right) \leq 0 \\
& \left.\operatorname{det}\left(\boldsymbol{T}+\boldsymbol{T}_{c}\right)=0, \operatorname{tr}\left(\boldsymbol{T}+\boldsymbol{T}_{c}\right) \geq 0\right\}, \\
& \mathcal{R}_{34}=\left\{\boldsymbol{T} \in \operatorname{Sym}: \operatorname{det}\left(\boldsymbol{T}-\boldsymbol{T}_{t}\right) \geq 0, \operatorname{tr}\left(\boldsymbol{T}-\boldsymbol{T}_{t}\right) \leq 0,\right. \\
& 2\left\|\boldsymbol{T}-\boldsymbol{T}_{s}\right\|^{2}-\left(1+\sin ^{2} \phi\right) \operatorname{tr}\left(\boldsymbol{T}-\boldsymbol{T}_{s}\right)^{2} \leq 0, \operatorname{tr}\left(\boldsymbol{T}-\boldsymbol{T}_{s}\right) \leq 0 \\
& \left.\operatorname{det}\left(\boldsymbol{T}+\boldsymbol{T}_{c}\right)=0, \operatorname{tr}\left(\boldsymbol{T}+\boldsymbol{T}_{c}\right) \geq 0\right\}, \\
& \mathcal{R}_{4}=\left\{\boldsymbol{T}_{c}\right\} .
\end{aligned}
$$

Let $\mathcal{E}_{i j}$ denote the region that will be mapped in $\mathcal{R}_{i j}$ and $\mathcal{E}_{0}$ the region that will be mapped in $\mathcal{R}_{0}$.

If $\mathbb{C} \boldsymbol{E} \in \mathcal{K}$ then $\boldsymbol{E} \in \mathcal{E}_{0}, \boldsymbol{T}=\mathbb{C} \boldsymbol{E}$ and $\boldsymbol{E}^{\boldsymbol{a}}=\mathbf{0}$. Otherwise, $\mathbb{C} \boldsymbol{E}$ has to be projected onto $\partial \mathcal{K}$, and $\boldsymbol{E}$ has to be splitted in two parts $\left(\boldsymbol{E}^{\boldsymbol{e}}+\boldsymbol{E}^{\boldsymbol{a}}\right)$ so that $\boldsymbol{T}(\in \partial \mathcal{K})=\mathbb{C}\left[\boldsymbol{E}-\boldsymbol{E}^{\boldsymbol{a}}\right]$.

Firstly, it can be directly verified if $\mathbf{E}$ belongs to one of the regions $\mathcal{E}_{0}, \mathcal{E}_{1}$ or $\mathcal{E}_{4}$

$$
\begin{array}{r}
\mathcal{E}_{0}=\left\{\boldsymbol{E} \in \mathrm{Sym}: \operatorname{det}\left(\mathbb{C} \boldsymbol{E}-\boldsymbol{T}_{t} \geq 0, \operatorname{tr}\left(\mathbb{C} \boldsymbol{E}-\boldsymbol{T}_{t} \leq 0,\right.\right.\right. \\
2\left\|\mathbb{C} \boldsymbol{E}-\boldsymbol{T}_{s}\right\|^{2}-\left(1+\sin ^{2} \phi\right) \operatorname{tr}\left(\mathbb{C} \boldsymbol{E}-\boldsymbol{T}_{s}\right)^{2} \leq 0, \operatorname{tr}\left(\mathbb{C} \boldsymbol{E}-\boldsymbol{T}_{s}\right) \leq 0 \\
\operatorname{det}\left(\mathbb{C} \boldsymbol{E}+\boldsymbol{T}_{c}\right) \geq 0, \operatorname{tr}\left(\mathbb{C} \boldsymbol{E}+\boldsymbol{T}_{c} \geq 0\right\},
\end{array}
$$




$$
\mathcal{E}_{1}=\left\{\boldsymbol{E} \in \mathrm{Sym}: \operatorname{det}\left(\boldsymbol{E}-\mathbb{C}^{-1} \boldsymbol{T}_{\boldsymbol{t}}\right)>0, \operatorname{tr}\left(\boldsymbol{E}-\mathbb{C}^{-1} \boldsymbol{T}_{\boldsymbol{t}}\right)>0\right\}
$$

or

$$
\mathcal{E}_{4}=\left\{\boldsymbol{E} \in \operatorname{Sym}: \operatorname{det}\left(\boldsymbol{E}+\mathbb{C}^{-1} \boldsymbol{T}_{\boldsymbol{c}}\right)>0, \operatorname{tr}\left(\boldsymbol{E}+\mathbb{C}^{-1} \boldsymbol{T}_{\boldsymbol{c}}\right)<0\right\} .
$$

If not, let us suppose that $\mathrm{E}$ belongs to $\mathcal{E}_{12}$. Let $\boldsymbol{f}_{1}, \boldsymbol{f}_{2}$ be the orthonormal basis of the characteristic space of $\boldsymbol{T}-\boldsymbol{T}_{t}$ and let $\theta \in[-\pi / 2, \pi / 2]$ be the unknown angle that $\boldsymbol{f}_{1}$ forms with $\boldsymbol{e}_{1}$ (Fig. 6), so that

$$
\boldsymbol{T}-\boldsymbol{T}_{t}=\sigma \boldsymbol{f}_{1} \otimes \boldsymbol{f}_{1} \quad \boldsymbol{N}^{12}=\boldsymbol{f}_{2} \otimes \boldsymbol{f}_{2}
$$

where $\sigma \leq 0$ is unknown.

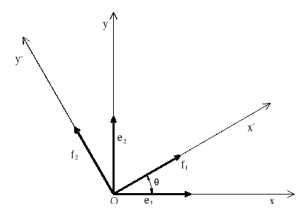

Figure 6: Reference systems.

Since

$$
\boldsymbol{f}_{1} \cdot \boldsymbol{e}_{1}=\boldsymbol{f}_{2} \cdot \boldsymbol{e}_{2}=\cos \theta, \quad \boldsymbol{f}_{1} \cdot \boldsymbol{e}_{2}=-\boldsymbol{f}_{2} \cdot \boldsymbol{e}_{1}=\sin \theta
$$

we obtain

$$
\begin{aligned}
& \boldsymbol{f}_{1} \otimes \boldsymbol{f}_{1}=\frac{1}{1+t^{2}}\left(\boldsymbol{e}_{1} \otimes \boldsymbol{e}_{1}+t\left(\boldsymbol{e}_{1} \otimes \boldsymbol{e}_{2}+\boldsymbol{e}_{2} \otimes \boldsymbol{e}_{1}\right)+t^{2} \boldsymbol{e}_{2} \otimes \boldsymbol{e}_{2}\right), \\
& \boldsymbol{f}_{2} \otimes \boldsymbol{f}_{2}=\frac{1}{1+t^{2}}\left(t^{2} \boldsymbol{e}_{1} \otimes \boldsymbol{e}_{1}-t\left(\boldsymbol{e}_{1} \otimes \boldsymbol{e}_{2}+\boldsymbol{e}_{2} \otimes \boldsymbol{e}_{1}\right)+\boldsymbol{e}_{2} \otimes \boldsymbol{e}_{2}\right),
\end{aligned}
$$

where $t=\tan \theta$.

The expressions of $\boldsymbol{T}$ and $\boldsymbol{N}^{12}$, with respect to the base $\boldsymbol{e}_{1}, \boldsymbol{e}_{2}$ can now be deduced by eqns (32)-(34), as functions of the unknowns quantity $\sigma$ and $t$, i.e.

$$
\begin{gathered}
\boldsymbol{T}=\frac{1}{1+t^{2}}\left(\sigma \boldsymbol{e}_{1} \otimes \boldsymbol{e}_{1}+\sigma t\left(\boldsymbol{e}_{1} \otimes \boldsymbol{e}_{2}+\boldsymbol{e}_{2} \otimes \boldsymbol{e}_{1}\right)+\sigma t^{2} \boldsymbol{e}_{2} \otimes \boldsymbol{e}_{2}\right) \\
+\sigma_{t x} \boldsymbol{e}_{1} \otimes \boldsymbol{e}_{1}+\sigma_{t y} \boldsymbol{e}_{2} \otimes \boldsymbol{e}_{2}+\tau_{t}\left(\mathbf{e}_{1} \otimes \mathbf{e}_{2}+\mathbf{e}_{2} \otimes \mathbf{e}_{1}\right), \\
\boldsymbol{N}_{12}=\frac{1}{1+t^{2}}\left(t^{2} \boldsymbol{e}_{1} \otimes \boldsymbol{e}_{1}-t\left(\boldsymbol{e}_{1} \otimes \boldsymbol{e}_{2}+\boldsymbol{e}_{2} \otimes \boldsymbol{e}_{1}\right)+\boldsymbol{e}_{2} \otimes \boldsymbol{e}_{2}\right) .
\end{gathered}
$$

Then, from the system $\boldsymbol{T}=\mathbb{C}\left[\boldsymbol{E}-\alpha \boldsymbol{N}^{12}\right]$, three equations can be obtained in the unknows $\alpha, \sigma$ and $t$, where $t$ is the solution of the algebraic equation:

$$
\begin{aligned}
& a t^{4}+b t^{3}+d t+e=0 \quad \text { with } \\
& a=E \phi \epsilon_{12}+\tau_{t}\left(\beta \nu^{2}-1\right), \\
& b=E\left(\phi \epsilon_{11}+\beta \epsilon_{22}\left(\beta \nu^{2}+\nu \phi-1\right)\right)+\left(\beta \nu^{2}-1\right)\left(\sigma_{t x}(\beta \nu+\phi)-\sigma_{t y}\right), \\
& d=-E \beta\left(\epsilon_{11}\left(\beta \nu^{2}+\nu \phi-1\right)+\epsilon_{22} \phi\right)-\left(1-\beta \nu^{2}\right)\left(\beta \sigma_{t x}-\sigma_{t y}(\beta \nu+\phi)\right), \\
& e=-\beta\left(E \phi \epsilon_{11}+\tau_{t}\left(\beta \nu^{2}-1\right)\right) .
\end{aligned}
$$


Once eqn (38) has been numerically solved,

$$
\alpha=\frac{\left(1+t^{2}\right)\left(E\left(\epsilon_{11}\left(t^{2}-\beta \nu\right)+\beta \epsilon_{22}\left(\nu t^{2}-1\right)\right)+\left(t^{2} \sigma_{t x}-\sigma_{t y}\right)\left(\beta \nu^{2}-1\right)\right)}{E\left(t^{4}-\beta\right)}
$$

and

$$
\sigma=\frac{\left(1+t^{2}\right)\left(E \beta\left(\epsilon_{11}-t^{2} \epsilon_{22}\right)-\beta \sigma_{t x}\left(1+\nu t^{2}\right)+\sigma_{t y}\left(t^{2}+\beta \nu\right)\right)}{\beta-t^{4}}
$$

can be determined. Now, if $\alpha \geq 0$ and $\sigma$ is such that $\boldsymbol{T}$, given by eqn (36), belongs to the intersection of $\mathcal{S}$ and $\mathcal{C}$ (see eqns (10), (11)), then $\boldsymbol{E} \in \mathcal{E}_{12}$ and $\boldsymbol{T}$ is the solution of the constitutive equation. If it does not happen, let us suppose $\boldsymbol{E}$ to belong to $\mathcal{E}_{23}$.

Let $\boldsymbol{g}_{1}, \boldsymbol{g}_{2}$ be the orthonormal basis of the characteristic space of $\boldsymbol{T}-\boldsymbol{T}_{s}$. Then,

$$
\begin{array}{r}
\boldsymbol{T}-\boldsymbol{T}_{s}=\sigma \boldsymbol{g}_{1} \otimes \boldsymbol{g}_{1}+\frac{\sigma(r-m)}{r+m} \boldsymbol{g}_{2} \otimes \boldsymbol{g}_{2}, \\
\boldsymbol{N}_{23}=\frac{\sqrt{2}(r-m)}{2 s} \boldsymbol{g}_{1} \otimes \boldsymbol{g}_{1}-\frac{\sqrt{2}(m+r)}{2 s} \boldsymbol{g}_{2} \otimes \boldsymbol{g}_{2},
\end{array}
$$

with $r=\sqrt{1+m^{2}}$ and $s=\sqrt{1+2 m^{2}}$. With respect to the orthonormal basis $\boldsymbol{e}_{1}, \boldsymbol{e}_{2}$, from eqns (41), (33) and (34), we obtain

$$
\begin{aligned}
\boldsymbol{T}= & \frac{-\sigma}{1+t^{2}}\left[\left(t^{2}-1-\frac{2 r t^{2}}{m+r}\right) \boldsymbol{e}_{1} \otimes \boldsymbol{e}_{1}-\frac{2 m t}{r+m}\left(\boldsymbol{e}_{1} \otimes \boldsymbol{e}_{2}+\boldsymbol{e}_{2} \otimes \boldsymbol{e}_{1}\right)\right. \\
& \left.+\left(1-t^{2}+\frac{2 r}{m+r}\right) \boldsymbol{e}_{2} \otimes \boldsymbol{e}_{2}\right]+\frac{\tau_{0 x}}{m} \boldsymbol{e}_{1} \otimes \boldsymbol{e}_{1}+\frac{\tau_{0 y}}{m} \boldsymbol{e}_{2} \otimes \boldsymbol{e}_{2} \\
& +\frac{\tau_{s}}{m}\left(\mathbf{e}_{1} \otimes \mathbf{e}_{2}+\mathbf{e}_{2} \otimes \mathbf{e}_{1}\right), \\
\boldsymbol{N}_{23}= & \frac{\sqrt{2}}{2 s\left(1+t^{2}\right)}\left[r\left(1-t^{2}\right)-m\left(1+t^{2}\right) \boldsymbol{e}_{1} \otimes \boldsymbol{e}_{1}+r t\left(\boldsymbol{e}_{1} \otimes \boldsymbol{e}_{2}+\boldsymbol{e}_{2} \otimes \boldsymbol{e}_{1}\right)\right. \\
& \left.\left.-m\left(1+t^{2}\right)-r\left(1-t^{2}\right) \boldsymbol{e}_{2} \otimes \boldsymbol{e}_{2}\right)\right]
\end{aligned}
$$

in the unknows $\sigma$ and $t$. Moreover, with the help of eqn (17), the unknows $\omega, \sigma$ and $t$ can be deduced from the three equations $\boldsymbol{T}=\mathbb{C}\left[\boldsymbol{E}-\omega \boldsymbol{N}^{23}\right]$ as in the previous region.

Now, if $\omega \geq 0$ and $\boldsymbol{T}$, as given by eqn (43), belongs to the intersection between $\mathcal{T}$ and $\mathcal{C}$ (see eqns (9), (10)), $\boldsymbol{E}$ belongs to $\mathcal{E}_{23}$ and $\boldsymbol{T}$ is the solution.

Otherwise, let us suppose $\boldsymbol{E}$ to belong to $\mathcal{E}_{2}$. If so, its image $\boldsymbol{T}$ has to belong to the intersection of $\mathcal{R}_{12}$ and $\mathcal{R}_{23}$. Then, $\boldsymbol{T}-\boldsymbol{T}_{t}$ can be write with respect to the orthonormal base $f_{1}, f_{2}$ as

$$
\boldsymbol{T}-\boldsymbol{T}_{t}=\sigma_{1} \boldsymbol{f}_{1} \otimes \boldsymbol{f}_{1}, \boldsymbol{N}^{12}=\boldsymbol{f}_{2} \otimes \boldsymbol{f}_{2},
$$

and, with respect to the base $\boldsymbol{g}_{1}, \boldsymbol{g}_{2}$ as

$$
\begin{array}{r}
\boldsymbol{T}-\boldsymbol{T}_{s}=\sigma_{2} \boldsymbol{g}_{1} \otimes \boldsymbol{g}_{1}+\frac{\sigma_{2}(r-m)}{r+m} \boldsymbol{g}_{2} \otimes \boldsymbol{g}_{2}, \\
\boldsymbol{N}_{23}=\frac{\sqrt{2}(r-m)}{2 s} \boldsymbol{g}_{1} \otimes \boldsymbol{g}_{1}-\frac{\sqrt{2}(m+r)}{2 s} \boldsymbol{g}_{2} \otimes \boldsymbol{g}_{2} .
\end{array}
$$


Let $\theta_{1}, \theta_{2} \in[-\pi / 2, \pi / 2]$ be the angles between $\boldsymbol{e}_{1}$ and, respectively, $\boldsymbol{f}_{1}, \boldsymbol{g}_{1}$. Then, from eqn (45)

$$
\begin{gathered}
\boldsymbol{T}=\frac{1}{1+t_{1}^{2}}\left(\sigma_{1} \boldsymbol{e}_{1} \otimes \boldsymbol{e}_{1}+\sigma_{1} t_{1}\left(\boldsymbol{e}_{1} \otimes \boldsymbol{e}_{2}+\boldsymbol{e}_{2} \otimes \boldsymbol{e}_{1}\right)+\sigma_{1} t_{1}^{2} \boldsymbol{e}_{2} \otimes \boldsymbol{e}_{2}\right) \\
\quad+\sigma_{t x} \boldsymbol{e}_{1} \otimes \boldsymbol{e}_{1}+\sigma_{t y} \boldsymbol{e}_{2} \otimes \boldsymbol{e}_{2}+\tau_{t}\left(\mathbf{e}_{1} \otimes \mathbf{e}_{2}+\mathbf{e}_{2} \otimes \mathbf{e}_{1}\right) \\
\boldsymbol{N}_{12}=\frac{1}{1+t_{1}^{2}}\left(t_{1}^{2} \boldsymbol{e}_{1} \otimes \boldsymbol{e}_{1}-t_{1}\left(\boldsymbol{e}_{1} \otimes \boldsymbol{e}_{2}+\boldsymbol{e}_{2} \otimes \boldsymbol{e}_{1}\right)+\boldsymbol{e}_{2} \otimes \boldsymbol{e}_{2}\right)
\end{gathered}
$$

and from eqn (46)

$$
\begin{aligned}
\boldsymbol{T}= & \frac{-\sigma_{2}}{1+t_{2}^{2}}\left[\left(\frac{2 r t_{2}^{2}}{-m-r}+t_{2}^{2}-1\right) \boldsymbol{e}_{1} \otimes \boldsymbol{e}_{1}+\frac{-2 m t_{2}}{r+m}\left(\boldsymbol{e}_{1} \otimes \boldsymbol{e}_{2}+\boldsymbol{e}_{2} \otimes \boldsymbol{e}_{1}\right)\right. \\
& \left.+\left(1-t_{2}^{2}-\frac{2 r}{-m-r}\right) \boldsymbol{e}_{2} \otimes \boldsymbol{e}_{2}\right]+\frac{\tau_{0 x}}{m} \boldsymbol{e}_{1} \otimes \boldsymbol{e}_{1}+\frac{\tau_{0 y}}{m} \boldsymbol{e}_{2} \otimes \boldsymbol{e}_{2} \\
& +\frac{\tau_{s}}{m}\left(\mathbf{e}_{1} \otimes \mathbf{e}_{2}+\mathbf{e}_{2} \otimes \mathbf{e}_{1}\right), \\
\boldsymbol{N}_{23}= & \frac{\sqrt{2}}{2 s\left(1+t_{2}^{2}\right)}\left[-m\left(1+t_{2}^{2}\right)+r\left(1-t_{2}^{2}\right) \boldsymbol{e}_{1} \otimes \boldsymbol{e}_{1}+r t_{2}\left(\boldsymbol{e}_{1} \otimes \boldsymbol{e}_{2}+\boldsymbol{e}_{2} \otimes \boldsymbol{e}_{1}\right)\right. \\
& \left.\left.-m\left(1+t_{2}^{2}\right)-r\left(1-t_{2}^{2}\right) \boldsymbol{e}_{2} \otimes \boldsymbol{e}_{2}\right)\right] .
\end{aligned}
$$

with $t_{1}=\tan \theta_{1}$ and $t_{2}=\tan \theta_{2}$. Therefore, by a comparison between eqns (47) and (50), we have

$$
\sigma_{1}=\frac{1+t_{1}^{2}}{m} \frac{m^{2} c_{1}+m\left(r c_{1}-c_{2}\right)+r c_{2}}{m\left(1-t_{1}^{2}\right)\left(1+t_{2}^{2}\right)-r\left(1+t_{1}^{2}\right)\left(1-t_{2}^{2}\right)},
$$

with $c_{1}=\sigma_{t x} t_{2}^{2}-\sigma_{t y}$ and $c_{2}=\tau_{0 y}-\tau_{0 x} t_{2}^{2}$,

$$
\sigma_{2}=\frac{1+t_{2}^{2}}{m} \frac{m^{2}\left(t_{1}^{2}-1\right)\left(t r T_{t}-t r T_{S}\right)+r\left(1+t_{1}^{2}\right)\left(\tau_{0 y}-\tau_{0 x}+m\left(\sigma_{t x}-\sigma_{t y}\right)\right)}{m\left(1-t_{1}^{2}\right)\left(1+t_{2}^{2}\right)-r\left(1+t_{1}^{2}\right)\left(1-t_{2}^{2}\right)}
$$

and

$$
\begin{aligned}
& m^{2}\left(\sigma_{t x} t_{2}\left(1-t_{1}\right)^{2}+\sigma_{t y}\left(t_{1}^{2} t_{2}+2 t_{1}-t_{2}\right)\right)+m\left(r t_{2}\left(\sigma_{t x}-\sigma_{t y}\right)\left(1+t_{1}^{2}\right)-t_{1}^{2} t_{2}\left(\tau_{0 x}+\tau_{0 y}\right)\right. \\
& \quad+2 t_{1}\left(t_{2}^{2} \tau_{0 x}-\tau_{0 y}+t_{2}\left(\tau_{0 x}+\tau_{0 y}\right)\right)+r t_{2}^{2}\left(1+t_{1}^{2}\right)\left(\tau_{0 y}-\tau_{0 x}\right)=0 .
\end{aligned}
$$

Moreover, as $\boldsymbol{E}^{a}$ is the linear combination $\boldsymbol{E}^{a}=\alpha N_{12}+\omega N_{23}$ and $\mathbb{C}\left[\boldsymbol{E}-\boldsymbol{E}^{a}\right]=\boldsymbol{T}$, three equations are deduced that, together with eqn (54), allow us to solve the problem in the unknowns $t_{1}, t_{2}, \alpha$ and $\omega$.

If $\alpha \geq 0, \omega \geq 0$ and $\boldsymbol{T} \in \mathcal{C}, \boldsymbol{E}$ belongs to $\mathcal{E}_{2}$ and $\boldsymbol{T}$ is its image in $\partial \mathcal{K}$. Otherwise, with a procedure similar to the previous one, the (unique) projection $\boldsymbol{T}$ of $\mathbb{C} \boldsymbol{E}$ onto $\mathcal{K}$ can be found.

\section{NUMERICAL EXAMPLE}

A very simple example is presented to highlight the potentials of the model, capable to accounting for the different directions material's strength. It takes into account solely the traction constraint and despite its simplicity, it allows to appreciate the capabilities of the proposed model generalization. The different directions material's stiffness have also been neglected since the focus is pointed out on material's strength. The results obtained via the 


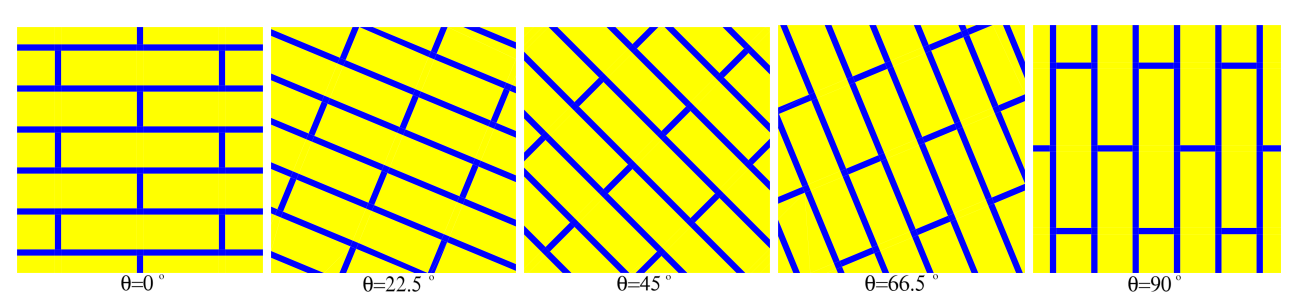

Figure 7: Samples considered in the numerical tensile tests.

proposed model is compared to those obtained by modelling at the micro-scale the texture of a brick's wall.

In the MADY code, the micro-scale models have been built by realizing suitable meshes and by assigning an isotropic non-linear elastic model to the mortar (blue) while the bricks (yellow) have been considered linear elastic - with $E=0.1 \mathrm{GPa}, \nu=0.1, \sigma_{t}=0.01 \mathrm{MPa}$, $\sigma_{c}=0.1 \mathrm{MPa}$ for the mortar, and $E=0.7 \mathrm{GPa}, \nu=0.1$ for bricks (see Fig. 7). These values of mechanical properties are usually used for mortar and bricks in masonry panels. It should however underline that the choice of suitable parameters values is beyond of the scope of the paper. In fact, the main purpose of the example presented is to highlight the model's capability of simulating the texture's effect in masonry structures. For the numerical simulations, 4nodes plane stress isoparametric elements have been used.

Five numerical tensile tests in the horizontal direction have been performed under displacement control, and the collapse load of the various samples has been determined.

For the "homogenous" model that no longer distinguishes between mortar and bricks, a Young modulus equal to that of the mortar has been assumed; as regards to the strength, the values of $\sigma_{t x}$ and $\sigma_{t y}$ have been calibrated by a comparison with the results of the test for $\theta=0^{\circ}$ and $\theta=90^{\circ}$. All the other cases have been modelled keeping unchanged the values of the mechanical parameters but assuming the appropriate rotations of $\mathcal{K}$. Thus, the generic value of $\mathbf{T}_{t}$ can be obtained as a function of $\sigma_{t x}, \sigma_{t y}$ and $\theta$, i.e.

$$
\begin{aligned}
\mathbf{T}_{t}= & \sigma_{t x} \cos ^{2} \theta+\sigma_{t y} \sin ^{2} \theta \mathbf{e}_{1} \otimes \mathbf{e}_{1}+\sigma_{t x} \sin ^{2} \theta+\sigma_{t y} \cos ^{2} \theta \mathbf{e}_{2} \otimes \mathbf{e}_{2} \\
& +\left(\sigma_{t y}-\sigma_{t x}\right) \sin \theta \cos \theta\left(\mathbf{e}_{1} \otimes \mathbf{e}_{2}+\mathbf{e}_{2} \otimes \mathbf{e}_{1}\right) .
\end{aligned}
$$

The graph of Fig. 8 shows the collapse stress vs $\theta$, as predicted by the proposed model compared to that obtained by means of the micro-scale tests.

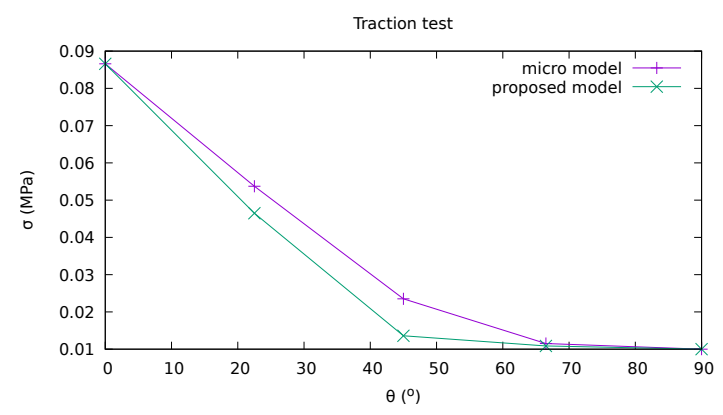

Figure 8: Collapse stress $\sigma$ vs $\theta$ as predicted by the proposed model and the micro-model. 


\section{CONCLUSION}

The proposed model allows to analyze plane bodies made of nonlinear elastic materials whose stress is forced to belong to the intersection of some closed and convex cones. In the proposed formulations, the vertices of the cones are allowed not to be spherical tensors. With this generalization, it has become possible to assign different strength in different directions. In this manner, the model is capable to capture some key aspects of the mechanical behavior of a masonry wall, that generally require to resort to more complex micro scale description. Nevertheless, given the lost of coaxiality between the stress and the inelastic strain becomes more difficult to solve the constitutive equation and some numerical escamotage are needed to have a efficient algorithm. Finally, the capabilities of differentiating the material's strength is a fundamental step of considering different damage process in different directions.

\section{REFERENCES}

[1] Del Piero, G., Constitutive equations and compatibility of the external loads for linear elastic masonry-like materials. Meccanica, 24, pp. 150-162, 1989.

[2] Lucchesi, M., Pintucchi, B. \& Zani, N., Masonry-like material with bounded shear stress. Eur. J. Mech. A Solids, 72, pp. 329-340, 2018.

[3] Lucchesi, M., Pintucchi, B. \& Zani, N., Bounded shear stress in masonry-like bodies. Meccanica 53(7), pp. 1777-1791, 2018.

[4] Lucchesi, M., Padovani, C., Pasquinelli, G. \& Zani, N., Masonry Constructions: Mechanical Models and Numerical Applications, Springer, 2008.

[5] Berto, L., Saetta, A., Scotta, R. \& Vitaliani, R., Shear behaviour of masonry panel: parametric FE analyses. International Journal of Solids and Structures, 41, pp. 43834405, 2004.

[6] Pelá, L., Cervera, M. \& Roca, P., Continuum damage model for orthotropic materials: Application to masonry. Computer Methods in Applied Mechanics and Engineering, 200(9-12), pp. 917-930, 2011.

[7] Lucchesi, M., Pintucchi, B. \& Zani, N., Orthotropic plane bodies with bounded tensile and compressive strength. Journal of Mechanics of Materials and Structures, 13(5), pp. 691-701, 2021.

[8] Lucchesi, M., Pintucchi, B. \& Zani, N., Intersection of convex cones as stress range for plane normal elastic bodies, Proceedings of 1st International Conference on Structural Damage Modelling and Assessment, 2021.

[9] Šilhavý, M., Mathematics of The Masonry-like Model and Limit Analysis, CISM Courses and Lectures, pp. 29-69, 2014.

[10] Mallik, P. K., Fiber-Reinforced Composites, M. Dekker, Inc.: New York, 1988. 